\title{
NEW THERAPIES IN UNRESECTABLE PANCREATIC NEUROENDOCRINE TUMORS
}

\author{
Ana-Georgiana Berescu ${ }^{1}$, Andreea-Mihaela Stoica ${ }^{1}$, Alexandra Bolocan ${ }^{1,2}$ \\ 'The University of Medicine and Pharmacy "Carol Davila", Bucharest, Romania \\ ${ }^{2}$ General Surgery and Emergency Clinic III - The University Emergency Hospital of Bucharest, Romania \\ Corresponding author: Ana-Georgiana Berescu \\ Phone no. 0040744963534 \\ E-mail: ana_berescu@yahoo.com
}

\begin{abstract}
Until recently, the main form of treatment for the pancreatic neuroendocrine tumors was the surgical resection (the Whipple operation). Due to technological advances, which resulted in more frequent diagnosis, attempts were made to discover other less radical forms of treatment, including limited surgical resection and multiple forms of chemotherapy. Surgical resection has shown benefit only on small lesions with no metastases. According to the recent studies chemotherapeutic agents have shown substantial benefits in terms of survival and disease progression, especially sunitinib and everolimus.
\end{abstract}

Keywords: pancreatic neuroendocrine tumors, chemotherapy, sunitinib, everolimus

\section{Introduction}

Pancreatic neuroendocrine tumors are rare cancers diagnosed less than 1,000 new cases per year in the USA. They represent between 3 to $5 \%$ of all pancreatic cancers and have a better prognosis than most forms of exocrine pancreatic tumors [1].

Pancreatic neuroendocrine tumors (PNT) derive from the islet cells of the pancreas (islets of Langerhans). These cells are responsible for the production and release of various hormones in the circulation. Pancreatic neuroendocrine tumors can be benign or malignant and can lead to overproduction of hormones, depending on the type of tumor, that give specific symptoms.

Pancreatic islet tumors may be functional or non-functional.

Non-functional tumors may produce substances that do not cause a specific symptomatology, the only symptoms are given by their growth and dissemination. They are generally malignant.

Functional tumors are the tumors that through producing hormones give a specific symptomatology. The main functional tumors are gastrinoma, insulinoma and glucagonoma.

Gastrinoma - characterized by the secretion of gastrin. Its increased secretion leads to stomach ulcers and diarrhea (Zollinger-Ellison syndrome). The common location is at the head of the pancreas. Most are malignant.

Insulinoma - Tumor that secretes insulin. They are usually slow growing tumors that do not disseminate. They can occur at any level of the pancreas. Most are benign.

Glucagonoma - secrete glucagon. It usually locates in the tail of the pancreas. Most are malignant. 
Other rare PNT: VIPoma (causes VernerMorrison syndrome) and somatostatinoma. These are listed in the same category due to their similar treatment [2,3].

Treatment options include surgical resection, chemotherapy and radiotherapy. Tumors that were detected in the early stages, before metastasis, benefit of surgical resection as long as the lesions are small and other comorbidities are not associated. With patients that associate liver metastases, an excision can be attempted. Radiotherapy is used as palliative treatment for symptoms relieve. Chemotherapy is strongly recommended for tumors in advanced stages that are inoperable [4].

\section{Discussions}

Despite progresses in treatment and diagnosis, many PNT are found in advanced stages, when surgery is no longer an option. Chemotherapy is not a curable treatment, only slowing the progression of the disease and increasing survival expectancy. For a better approach to PNT cases, several studies are in progress.

Before 2011, the only chemotherapeutic agent approved for the PNT was Streptozocin that despite its efficiency proved to be very toxic. Streptozotocina is similar enough to glucose, thus transported into the cells by glucose transport protein GLUT2, only it is not recognized by other glucose transporters. This explains its toxicity to beta cells because these cells have a relatively high level of GLUT2. Recently new chemotherapies were tested, CAPTEM Regimen, (Sunitinib and Everolimus) was noted as a better alternative [5].

To evaluate the response to chemotherapy, in 2000, an international commission established a unique criterion, easy to apply, known as RECIST. They allow the measurement of tumor response using X-ray, CT scan and MRI. They are based on a simplification of the former methods (WHO, ECOG) and they require the presence of at least one measurable lesion shown by a imagistic method.

RECIST registers four response categories:

- CR (complete response) $=$ disappearance of all target lesions
- PR (partial response) $=30 \%$ decrease in the sum of the longest diameter of target lesions

- PD (progressive disease) $=20 \%$ increase in the sum of the longest diameter of target lesions

- SD (stable disease) = small changes that do not meet above criteria [6]

Depending on the disease's response to treatment, it will be further placed under one of the listed categories. RECIST has been widely accepted as a standardized measurement of tumoral response being updated regularly [6-8].

\section{CAPTEM regimen}

Previously used chemotherapy schemes (streptozocin) have many side effects, the worst being their toxicity. Several studies have been conducted in order to review the association between temozolomide and other chemotherapeutic agents, the best result was given by the association with capecitabina. Temozolomide (sometimes called TMZ) is an imidazotetrazine derivative of the alkylating agent dacarbazime that undergoes rapid chemical conversion in the systemic circulation at physiological $\mathrm{pH}$ to the active compound (MITC), thus interfering with DNA replication. Capecitabine is a pro-drug, enzymatically converted to 5-fluorouracil in the body, which further is an inhibitor of thymidylate synthase, the enzyme that catalyzes the formation of thymidine monophosphate (active form of thymidine) required for de novo synthesis of DNA and RNA during gene expression [9]. Based on the information obtained from these studies, Saif et al. [10] have developed a new regimen called CAPTEM (capecitabine + temozolomide). The objective of the study that was conducted between 2006 and 2013, was to evaluate the efficiency and safety of this regimen. The study was conducted on 7 patients (4 men and 3 women) diagnosed with progressive metastatic PNT that have not responded to previous chemotherapy treatments applied on average for two years, including doxorubicin, streptozocin, cisplatin and gemcitabine. They were treated with capecitabine at a flat dose of $1,000 \mathrm{mg}$ orally t.i.d. on days 1-14 and temozolomide 200 $\mathrm{mg} / \mathrm{m}^{2}$ was given in two divided doses daily on days $10-14$ of a 28 -day cycle. After each cycle, 
serum tumor markers were measured and every two cycles were evaluated imagistically. The response of each patient was evaluated and classified according to the level of toxicity and RECIST criteria, showing a 3-4 toxicity level (most commonly were recorded: neutropenia, fatigue, hand-foot syndrome).The clinical benefit was $71 \%$ ( 3 patients PR and 2 patients $\mathrm{SD})$. The mean survival time was 24 months but 3 patients were still alive at the time of the study report. The overall benefit of the CAPTEM regime was proven to be its toxicity profile. CAPTEM proved to be a well-tolerated oral treatment with a good safety profile. Further studies are required in order to compare CAPTEM regime to temozolamide in monotherapy.

\section{Everolimus}

Molecular biology studies on PNT allowed the discovery and approval of new therapies including the oral mTOR inhibitor, Everolimus. Everolimus blocks the signal transduction in tumor cells and cells that play a role in tumor angiogenesis. Yao et al. reviewed published data on two Phase II clinical trials and a Phase III trial on the use of oral everolimus in advanced PNT.

In the first study (University of Texas MD Anderson Cancer Center phase II study) [11], patients with advanced PNT, progressive or not, were treated with LAR octreotide $30 \mathrm{mg}$ intramuscularly and 5 or $10 \mathrm{mg} /$ day oral everolimus. Patients were monitored by CT or MRI every 12 weeks, showing a better response rate and an average progression-free survival of 11.6 months in patients receiving $10 \mathrm{mg} /$ day everolimus.

The second phase II trial (RADIANT-1) [12] included patients with advanced PNT and progressive disease that were treated with everolimus in monotherapy or the combination everolimus-octreotide. Results showed an increase in mean survival for combined therapy (16.7 months) compared to monotherapy (9.7 months).

In the Phase III trial (RADIANT-3) [13] patients with PNT received randomly everolimus or placebo. Mean survival was 11 months for the administration of everolimus and only 4.6 months for placebo. Also, everolimus significantly reduced hormonal secretion of functional PNT and the markers associated with angiogenesis (VEGF soluble receptor 2 and placental growth hormone) [14].

Data reviewed by Yao et al. suggest that treatment with everolimus delays tumor progression and should be considered in the treatment of patients with advanced PNT.

\section{Sunitinib}

Tyrosine kinase receptors have an important role in controlling cell proliferation in the PNT. Sunitinib is an oral tyrosine kinase inhibitor that blocks the activation of angiogenesis and stops cell proliferation [15]. It has already been approved in several countries for the treatment of unresectable or metastatic PNT.

In vitro, it was shown that sunitinib inhibits cell growth stimulated by VEGF, KIT, PDGF, RET, CSF-1; in studies on mice it was observed a significant reduction of endothelial cell density and tumor vessel pericytes layer (Delbaldo et al., 2012) [16] and also a significant reduction of the vascularization and tumor volume (Olson et al., 2011) [17].

Several studies have been conducted with sunitinib at doses between 50 to $150 \mathrm{mg}$ / day in order to establish the safety, pharmacokinetics and recommended dose. The maximum tolerated dose was $75 \mathrm{mg} /$ day and as adverse reactions occurred fatigue, high blood pressure and bullous skin lesions, reversible at the end of the treatment. Therefore, the recommended dose is $50 \mathrm{mg} / \mathrm{day}$; side effects that may occur are edema and neutropenia. Also at doses $\geq 50 \mathrm{mg} /$ day, hair discoloration and the yellowing of the skin could be observed.

In another study conducted on 107 people with advanced carcinoid and PNT (carcinoid = 41; neurendocrine pancreatic tumors $=66$ ) patients received sunitinib in repetitive 6-week cycles $(50 \mathrm{mg} /$ day orally for 4 weeks, followed by 2 weeks without treatment). The response rate of patients with PNT was $16.7 \%$, and in $68 \%$ the disease remained stable (SD) [18].

Sunitinib has shown efficiency in numerous clinical trials, helping to maintain the quality of life for people with PNT. 


\section{Conclusions}

Pancreatic neuroendocrine tumors, considered rare tumors, show a rising incidence, probably due to the technological developments that allow more frequent diagnosis. Due to the increase in incidence, it was considered necessary to develop new treatment options. The approval of everolimus and sunitinib is an important step in the medical field that marks the evolution of targeted therapies. Further research and the development of new therapies will improve not only the treatment, but also the quality of life for people diagnosed with this disease.

\section{References}

[1]Bethesda, MD. National Cancer Institute: $P D Q{ }^{\circledR}$ Pancreatic Neuroendocrine Tumors (Islet Cell Tumors) Treatment. 2014 Jul. Available at: http://www.cancer.gov/cancertopics/pdq/treatment/is letcell/HealthProfessional/page1.

[2]Bethesda, MD. National Cancer Institute: $P D Q{ }^{\circledR}$ Pancreatic Neuroendocrine Tumors (Islet Cell Tumors) Treatment. 2014 Aug. Available at: http://cancer.gov/cancertopics/pdq/treatment/isletcell /Patient.

[3]Goldfinger SE, Strosberg JR. Classification, epidemiology, clinical presentation, localization, and staging of pancreatic neuroendocrine tumors (isletcell tumors). 2014 Jun. Available from: http://www.uptodate.com/contents/classificationepidemiology-clinical-presentation-localization-andstaging-of-pancreatic-neuroendocrine-tumors-isletcell-tumors.

[4]Cummins M, Pavlakis N. The use of targeted therapies in pancreatic neuroendocrine tumours: patient assessment, treatment administration, and management of adverse events. Ther Adv Med Oncol. 2013;5(5):286-300.

[5]Oberstein PE, Saif MW. Update on novel therapies for pancreatic neuroendocrine tumors. Jop. 2012;13(4):372-375.

[6]Imaging Response Criteria. National Cancer Institute. Available from: http://imaging.cancer.gov/clinicaltrials/imaging.

[7]Nishino M, Jackman DM, Hatabu H, et al. New Response Evaluation Criteria in Solid Tumors (RECIST) guidelines for advanced non-small cell lung cancer: comparison with original RECIST and impact on assessment of tumor response to targeted therapy. AJR Am J Roentgenol. 2010;195(3):W221228.

[8]Eisenhauer EA, Therasse P, Bogaerts $\mathrm{J}$, et al. New response evaluation criteria in solid tumours: revised RECIST guideline (version 1.1). Eur J Cancer. 2009;45(2):228-247.

[9]Xeloda (capecitabine) dosing, indications, interactions, adverse effects, and more. Medscape Reference. WebMD. 25 January 2014.

[10]Saif MW, Kaley K, Brennan M, Garcon MC, Rodriguez G, Rodriguez T. A Retrospective Study of Capecitabine/Temozolomide (CAPTEM) Regimen in the Treatment of Metastatic Pancreatic Neuroendocrine Tumors (pNETs) after Failing Previous Therapy. Jop. 2013;14(5):498-501.

[11]Yao JC, Phan AT, Chang DZ, Wolff RA, Hess K, Gupta S, et al. Efficacy of RAD001 (everolimus) and octreotide LAR in advanced low- to intermediate-grade neuroendocrine tumors: results of a phase II study. J Clin Oncol 2008;26:4311-8.

[12]Yao JC, Lombard-Bohas C, Baudin E, Kvols LK, Rougier P, Ruszniewski P, et al. Daily oral everolimus activity in patients with metastatic pancreatic neuroendocrine tumors after failure of cytotoxic chemotherapy: a phase II trial. J Clin Oncol 2010;28:69-76.

[13]Yao JC, Shah MH, Ito T, Bohas CL, Wolin EM, Van Cutsem E, et al. Everolimus for advanced pancreatic neuroendocrine tumors. N Engl J Med 2011;364:514-23.

[14]Yao JC, Tsuchihashi Z, Panneerselvam A, Winkler RE, Bugarini R, Pavel M. Effect of everolimus treatment on markers of angiogenesis in patients with advanced pancreatic neuroendocrine tumours (pNET) - results from the phase III RADIANT-3 study. Eur J Cancer 2011;47 Suppl 1:S463.

[15]Faivre S, Delbaldo C, Vera K, et al. Safety, pharmacokinetic, and antitumor activity of SU11248, a novel oral multitarget tyrosine kinase inhibitor, in patients with cancer. J Clin Oncol. 2006;24(1):2535.

[16]Delbaldo C., Faivre S., Dreyer C., Raymond E. (2012) Sunitinib in advanced pancreatic neuroendocrine tumors: latest evidence and clinical potential. Ther Adv Med Oncol 2012;4: 9-18.

[17]Olson P., Chu G., Perry S., Nolan-Stevaux O., Hanahan D. (2011) Imaging guided trials of the angiogenesis inhibitor sunitinib in mouse models predict efficacy in pancreatic neuroendocrine but not ductal carcinoma. Proc Natl Acad Sci U S A;108: E1275-E1284.

[18]Kulke MH, Lenz HJ, Meropol NJ, et al. Activity of sunitinib in patients with advanced neuroendocrine tumors. J Clin Oncol. 2008;26(20):3403-10. 